\title{
Engaging Autistic Children in Imitation and Turn-Taking Games with Multiagent System of Interactive Lighting Blocks
}

\author{
Jeroen C. J. Brok and Emilia I. Barakova \\ Eindhoven University of Technology \\ ID, Den Dolech 2, POB 513, \\ e.i.barakova@tue.nl
}

\begin{abstract}
In this paper game scenarios that aim to establish elements of cooperative play such as imitation and turn taking between children with autism and a caregiver are investigated. Multiagent system of interactive blocks is used to facilitate the games. The training elements include verbal description followed by imitation of video-modeled play episodes. By combining this method with the tangible multiagent platform of interactive blocks (i-blocks) children with autism could imitate play episodes that involved turn taking with a caregiver. The experiment showed that most of the children managed to imitate the play scenarios after video modeling, and repeat the behaviors with the tangible and appealing block platform. When all the actions were well understood by the autistic children, they performed willingly turn taking cooperative behaviors, which they normally do not do.
\end{abstract}

Keywords: Games, collaborative play, tangibles, i-blocks, autism, behavioral training, turn taking, imitation.

\section{Introduction}

Teaching of socially relevant behavior to children through games with robotic toys is an area of emerging interest [3, 4, 6, 9, 21, 25]. By robotic toys we understand autonomous robots and tangibles that are equipped with sensors and actuators, mediated by a computational device. They can perceive and act upon the surrounding environment that may include other robotic toys or humans and in this way they have the affordance to be used as a mediator of play and education [3, 4, 6, 9, 21, 24, 25, 26].

A number of robotic toys, both with and without anthropomorphic or zoomorphic shape have been used in games with autistic children $[5,7,9,20,21,22,25,26]$. The autonomous robotic toys in these studies, perform simple behavior (as for instance lifting of a hand) that aims to provoke reciprocal human reaction $[9,10,21]$, to predict the position or the direction of the movement which is referred to as predicting motor intentions [5, 9, 21, 29], and become a mediator of play [3, 6, 26].

Robotic toys have been shown to engage children with autism at various age levels $[3,16,25,27]$ because of their predictable and controlled behavior, their physical embodiment and because of the appeal that computerized games have to people with autism. Tangibles, in particular, have been shown to support collaboration by typical 
children [8, 17]. Experiments with "Lego Therapy" [19, 23] suggest that interaction with physical building blocks may support autistic children to collaborate for extended periods of time. In discussions with caregivers in different autistic institutions we found out that it is a common practice to turn abstract concepts like time and mood to visible or tangible categories, by using for instance adapted clocks for visualization of the time and traffic light to express moods through colors.

We use a multiagent platform of interactive blocks (i-blocks) that express behavior in accordance with the way the blocks are used [2, 3, 4]. The platform has a resemblance with tangible interaction platform, because of its appearance, and with a multiagent system of robots, since it expresses behavior through changing the lighting pattern of the blocks in emergent manner [4]. It has shown to be very engaging for children with autism, because of the following reasons. Blocks are a usual toy for children with autism. The regular cubic shape enables the children to form a grid in order to make the spatial relations more obvious. In addition, the cubes can be stacked, enabling more complex structures. The blocks that we have developed emit dimmed colored lights which change in accordance with the way the blocks are used. Light stimulation is by itself known to be appealing to the autistic children. In addition, the puzzling lighting behavior motivates the autistic children to find out the underlying rule of interaction of the blocks and to explore with the platform in order to discover new emergent behaviors.

Nadel [21] showed, that imitation, turn taking, and recognition of mutual action patterns is a basis for developing the sociall skills and inferring others' intention correctly. Inspired by these findings, in the current study we use the i-blocks platform for turn taking and imitation games.

Earlier research indicated that if the children with autism are asked to make a verbal description of the acted out target behavior before imitating it helped them to sustain cooperative play, which resulted in longer play episodes and more variation in play [18]. It was shown that explaining beforehand the performed behavior to the autistic child makes possible more complex imitation to be performed. Different studies have established that autistic children would imitate either the goal of the imitation, or the particullar movement behavior. Combining both, however have shown to be challenging for the children with autism.

Therefore we prepared scenarios that contained relatively complex imitation behaviors in which the children had to understand, verbalize, and imitate these behaviors. Similar to Jahr and colleagues [18], we used a person to explain the behavior that has to be imitated to the children. Inspired by studies of videomodelling, such as the ones reported in $[11,12,13,14,28]$, instead of using actors or the same individual to show the behavioral scenarios every time, we made videos of the demonstrated scenarios.

We developed cooperative play scenarios that involved imitation and turn taking between children with autism and a caregiver. In our approach to training children with autism, we target the possible complex of reasons that may cause the atypical or missing cooperative play. First, we address the lack in variation during play that makes the child's development sometimes one-sided and limits social interaction with peers. Second, we also consider the difficulties of autistic children to stay concentrated and keep their attention to the current activity. Third, we focus on imitation of the targeted behavior, instead of the individual movements. 


\section{Training Social Play Behaviors by Autistic Children.}

In an investigation of social play behavior Parten [24] distinguishes between several types of play, depending on the level of social involvement of the children, namely solitary independent play, parallel activity, associative play, and cooperative or organized supplementary play. These play behaviors were found typical for different age groups.

In solitary independent play and parallel activity the child plays independently from other children. By parallel activity the child is situated among other children and plays with toys that are like those which the other children are using, but does not try to influence/modify the activity of the nearby children. In associative and collaborative play the child plays with other children. There is a greater level of awareness of the peers and there is a borrowing and loaning of play material; there is evident interaction, as for instance, following one another with trains or wagons. By collaborative play there is clear exchange of play objects.

Autistic children are most often observed to be engaged in solitary independent play and some in parallel activity, even when their age progresses. We aim to train elements of associative or collaborative play through appropriate games. Problems in the social aspects of play in autism can be traced to the earliest forms of social interaction with the caregivers and may include a range of behaviors. We consider imitation behavior as central to social learning and development.

Imitation does not occur naturally in social development of people with autism. Therefore, imitation behaviors have been used in many different ways to help autistic children improve their social skills. For instance, children with autism do not imitate naturally but like being imitated. A typical experimental scenario requires a caregiver to imitate the autistic child in order to engage him/her in social interaction $[15,17]$. Aldridge and coleagues [1] conducted experiments with autistic people that aim to differentiate the elements of imitation that are not deficient by autistic people. They constructed an experiment with 'unfulfilled intentions' tasks and found, much to their surprise, that the autistic children (mean age $=39$ months) did imitate the goal of an action very well. Another studies showed that although the children can imitate the goal of an action, they would either fail on mimicry, i.e. repeating the actions, if the relation between those actions and the outcome they produce is not clear, or they fail by imitation proper, i.e. the imitation of both: the means and the goal of an action.

We designed an experiment where the goal of the imitation has to be achieved through performing a sequence of simple turning movement behaviors.

Next to imitation, facilitating turn taking is particularly important to the children with autism because of their weak social responsiveness. Sensitivity to the contingencies involved in cooperative play may produce turn taking and variability in the partners play behavior.

To address the problem of turn taking through the imitation of the goal of an action, we used a task similar to those of Jahr [18] where actors were demonstrating actions to the children. To minimize the stress levels of newnes due to meeting actors that can behave differently every time we asked a single actor to demonstrate all the scenarios. Both, the request for social interaction and the newness of the actor can make it unnecesary stressfull for the child to attend the training. To further resuce this 
stress, we created video scenarios instead of using physically present actor, similar to Charlop-Christ and colleagues [11] .

In summary, our approach is expected to have the following advantages. (1) The same actor can do every scenario, which allows the child to develop a bound with the actor. (2) The child won't feel as stressed watching a video as he or she will be while watching live acted scenarios. There is no social response expected and therefore the child will be more relaxed. (3) The tape can be distributed anywhere. (4) One can focus more on the important aspects in the video, by zooming in at highlights or blurring out unwanted stimuli. This will keep the child from getting distracted by unimportant details. (5) Video modeling will also allow the actors to shoot an optimal scenario. (6) There will be less people required to provide the necessary support for the children.

\section{Experimental Design}

\subsection{Experimental Procedure}

The experimental procedure contains out of three steps that are repeated every time. (1) The autistic child watches the video of the target behavior together with a coordinator. The coordinator will make sure the child pays attention while the actors in the video play out the scenario step by step. (2) The coordinator lets the child describe what was shown in the video scenario. This procedure has to be repeated until the child describes the whole scenario without errors. (3) The coordinator will than imitate the scenario together with the child. After completion of the scenario without errors the video with increased complexity (for instance increased number of play responses) can be presented.

Teaching autistic children to imitate at a very early age can be crucial for their development. Therefore we target very young children. As shown by [18] two important requirements considering the children have to be satisfied. (1) The child has to be able to follow instructions and perform play responses with toys upon verbal instructions. (2) The child has to be able to describe play activities. After watching the video the child has to be able to describe what he or she has seen.

The quality of the video might also influence the results. Earlier research [17] has shown that it is not important whether the actors are adults or children. However, the quality of the act is essential. The actors have to be trained to perform a list of scripted play episodes. This list has to be assessed by an expert to define whether this is an appropriate play scenario. The first video introduced the blocks, the 3 interaction possibilities, and a play scenario for the child to practice individually. The second video was a scenario with two play responses; the child imitated one action from the scenario than shared his play area and let the other person complete the scenario. The final video showed four play responses which required an additional turn taking behavior than the second video.

\subsection{The Multiagent Platform of Interactive Blocks -i-Blocks}

We use multiagent system of i-blocks [2, 3, 4], which interact when positioned in each other's vicinity (Figure 1). They emit colored light and depending on the algorithm that 


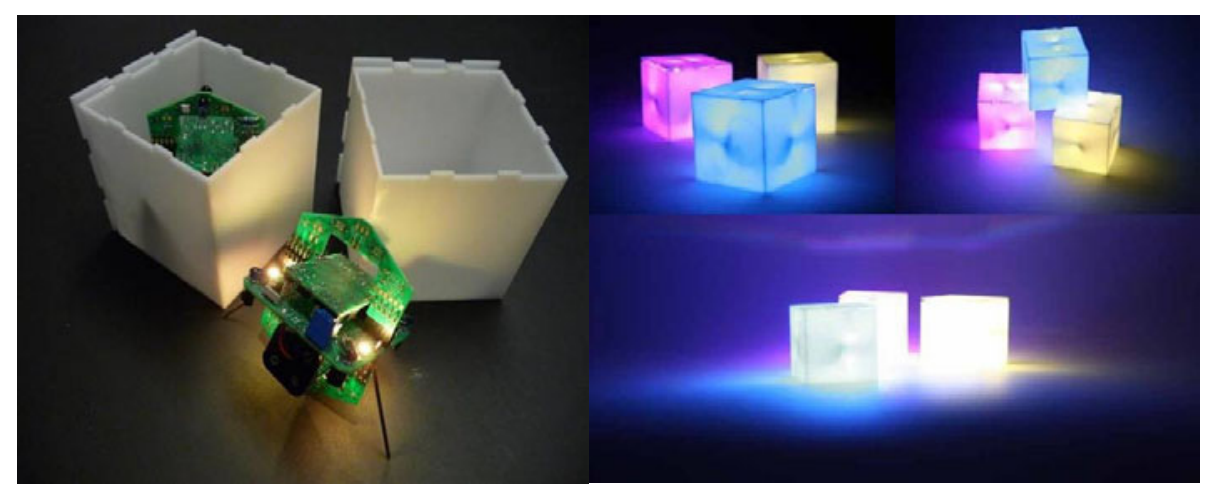

Fig. 1. The developed multi-agent system of interactive blocks (a) internals of the block platform and (b) example of emergent light patterns when the blocks are put in each other's vicinity

is loaded on each block at this moment, express different set of local interaction behaviors that cause emergent collective behaviors. The overall behavior of the system depends on the local interactions, and therefore it forms an embodied multiagent system [4]. The complexity of the emergent behaviors depends on the complexity of the individual behaviors of the blocks.

We intended to create games that are challenging, but still not too unpredictable that the child wouldn't understand the behavior. Cooperative play can be assessed by the number of play responses. By encouraging turn taking and motivating the players to work towards achieving a common goal, we assume that the play responses will increase.

There was one active block (which can change the colors of other blocks) and 5 passive blocks, that can be subject to a color change. There are sheets of colored paper at the 4 corners of the table. The task for the children was to match the color of the blocks to the pieces of colored paper positioned in front of the children. For example a colorless passive receiving block laying on red piece of paper should be colored red. This can be done by turning the active sending block to red and holding it close to the passive receiving block. Turning the active block causes a change of its color and this is the behavior that the child has to imitate.

Examples of different behavioral possibilities of the blocks in the framework of different game scenarios are described in [2, 3, 4]. In [2] the technical features of the platform are described. The blocks can interact by detecting their neighbors through the built-in sensors, and show behavior in response to their sensory stimulation. A build-in accelerometer is used to record the children's hand movement and to facilitate the imitation game needed for the current study.

The blocks were specially designed to fit the play habits and the patterns of thinking of the autistic children. Initial user tests as reported in $[3,4]$ have shown that children find them very engaging and pleasurable.

\subsection{Play Scenarios}

Three scenarios has been performed and tested in the following way. First, the child and the coordinator view the video scenario on a computer or TV (TV offers less 
distractions; computer keyboard and mouse distract the child). After that the coordinator asks the child to describe what actions were performed by the actor. If the scenario is described correctly the child can start imitating the scenario, if not the video scenario is re-played until the child explains correctly the actions. The child plays the scenario with the coordinator.

Scenario one: The blocks are placed in front of the actor, the passive blocks are on the colored pieces of paper, and the active block is in the middle. The actor picks up the active block and starts turning it until the block gets the color of the paper in front of him. The actor transfers the color to the passive block on the piece of paper. $\mathrm{He}$ does so for all the three paper-block combinations. The scenario is finished when all passive blocks are colored according to the underlying piece of paper.

Goal: The child gets familiar with the interaction possibilities of the blocks and the concept of the game.

Implementation: The blocks are now placed in front of the child. The child has to perform the following actions:

1.1 Child watches video

1.2 Child describes scenario

1.3 Child colors first block

1.4 Child colors second block

1.5 Child colors third block

The game is finished when all passive blocks are colored according to the underlying piece of paper.

Scenario two: In this scenario the actions were performed by two actors. The blocks are placed between the two actors. The first actor picks up the active block and starts turning it until the block becomes the right color. He transfers the color to the passive block on the piece of paper. The first actor shares the block with the second actor who, on his turn, colors his block.

Goal: let the child complete the most basic cooperative scenario, where the child shares the block once with the coordinator.

Implementation: After the video the coordinator asks the child what actions were performed by the two actors, and how they responded to each other. The blocks are now placed between the child and the coordinator. The following actions take place:

2.1 Child watches video

2.2 Child describes scenario

2.3 Child colors first block

2.4 Child shares block (cooperates)

2.5 Coordinator colors a block

The game is finished when the child shares the block with the coordinator who colors his or her block in the same way.

Scenario three: This is an extension of Scenario two where the active block is shared twice.

Goal: let the child complete a longer scenario, where he/she shares the block two times and receives the block once. 


\section{Implementation:}

3.1 Child watches video

3.2 Child describes scenario

2.3 Child colors first block

3.4 Child shares block (cooperates)

3.5 Coordinator colors block

3.6 Coordinator shares

3.7 Child colors $2^{\text {nd }}$ block

3.8 Child shares block (cooperates)

3.9 Coordinator colors $2^{\text {nd }}$ block

The scenario ends after the coordinator colored his or her second block.

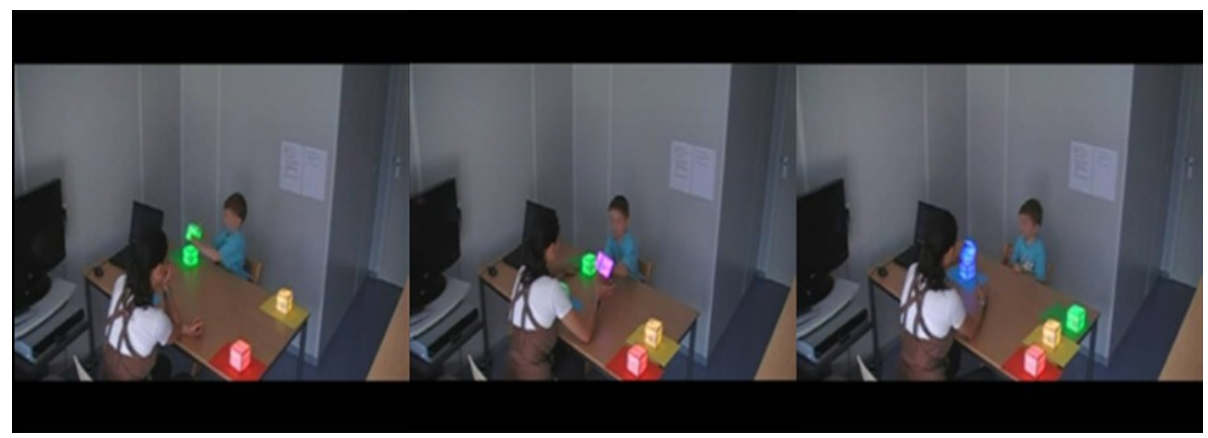

Fig. 2. Snapshots of performing scenario 3. a) Child shares block (cooperates) b) Coordinator colors $2^{\text {nd }}$ block after the second turn taking.

\section{Results}

\subsection{User Observations}

To verify the experimental choices, we conducted a confrontation of autistic children with the platform and the experimental conditions. All the participants were aged between six and eight. They were diagnosed with some form of autism. The first two participants didn't see the introducing video, but were allowed to explore the behavior of the blocks together just to find out whether the introduction video was necessary. The children enjoyed the free playing, but didn't manage to perform scenarios 2 and 3. Participant 1 (P1) found experimenting far more interesting than watching the videos. He was constantly distracted from the video by playing with the blocks. Although P1 got distracted several times during the video he and P2 were able to complete the scenarios. The rest of the children played together with the coordinator instead of playing with peers.

P3 had no difficulties understanding the games and even figured out the final game before the video was completely finished. P4 was very focused while watching the video scenarios. After fully describing the games P4 imitated them perfectly without any mistakes. P5 was very involved in the videos; he counted the blocks together with the actors in the videos and named the colors like the actors did. He was not only very 
involved, but also playful and took initiative to explore. After completing the scenarios without any problems P5 started to build towers and explore the blocks interactive possibilities. P6's comments and repetitive behavior gave the impression that she tries to remember colors by establishing a pattern. P6 was the only one who needed to rewatch the scenarios about turn taking to complete them without mistakes.

The scenarios are in general too easy for children of this age group and therefore should be either tested with younger children or made more difficult and challenging for this group. As seen in Table 1 the blocks brought a high level of distraction for the children while they were watching the scenarios.

Table 1. The number of distractions per participant

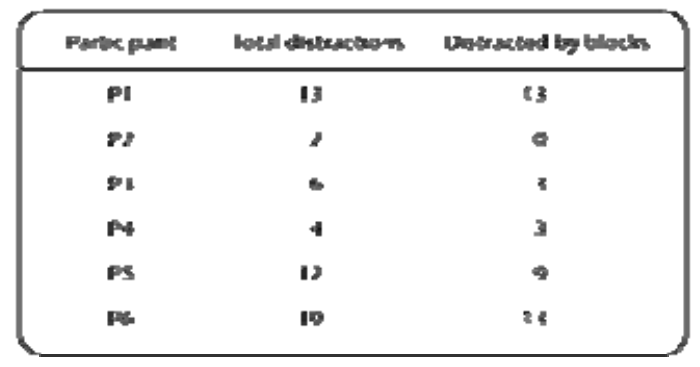

Another important finding was that it is hard to make the children exactly describe the actions. The child possibly has to be informed before the video starts that he or she will have to describe the actions in the video. In the current setting the children often already started playing during the video or directly after it.

\subsection{User Test}

The user test was performed with five autistic children of 4 and 5 years of age. Two of them were diagnosed with PDD-NOS (P1,P2), one PDD-NOS or classic autism (P3), one classic autism and ADHD (P4) and for the fifth child there were no definite outcomes of the diagnosis yet (P5). All the children managed to finish two or three of the scenarios.

The children were prepared for the test by their development coordinator, who also guided the children from their classroom to the test room and imitated the scenarios with the children. At the end she wrote a qualitative evaluation of the tests which will be summarized below.

P1 didn't have much trouble with the games. He finished all three scenarios without any errors. There was only one short distraction when the child looked up from the third video to check out the blocks. The rest of the videos were watched without any distractions and without any necessary encouragement from the coordinator.

P2 was very enthusiastic about playing with the blocks, despite he normally does not show variation in play. He wanted to start playing right away, but after calming down he watched the videos without more than one distraction moment looking over at the blocks. An interesting observation was that P2 who normally has trouble sustaining one on one play, was now concentrated on the scenarios and followed the 
instructions. During scenario three he first didn't shared the block with the coordinator, but eventually after asking what he had seen in the video he completed it by involving the coordinator in the play scenario.

P3 usually doesn't show initiatives in play, he is very passive and has to be stimulated a lot. Watching videos was one of his favorite activities and therefore he became enthusiastic for our scenarios very quickly. His approach during the session was more active than normally in the group, he counted together with the actress in the video and mentioned the colors like she did. The final scenario was completed after watching the video second time because the first time the imitation that $\mathrm{P} 3$ showed was not correct.

P4 normally shows minimal group play and play variability - he plays every day with one and the same train. It took more effort to get him to participate in the session. The child had to be stimulated several times to keep watching the video. It was surprising that he followed the instructions and let the coordinator join in the game. The last most difficult scenario was too hard for him. After watching the video for the second time the child didn't manage to complete the scenario, because he became heavily distracted.

P5 already shows quite varied play, but still had turn taking problems. He was very enthused and explorative with the blocks. He was focused on the videos and showed turn taking during all the scenarios without needing further instructions or visualization.

In Table 2 the number of distractions in the test are shown. Although the total distraction still varies widely per person the distractions by the blocks considerably decreased because of the better preparation of the test. The total number of distractions also went down due to better preparation of the coordinator.

Table 2. The number of distractions per participant.- improved experimental design

\begin{tabular}{|c|c|c|}
\hline Pantionat & 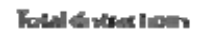 & Ontand by both \\
\hline FI & ' & 2 \\
\hline $\mathbf{F z}$ & I & o \\
\hline P3 & $y$ & 0 \\
\hline w & 21 & $\mathbf{a}$ \\
\hline $\mathbf{p s}$ & I & n \\
\hline
\end{tabular}

\section{Discussion}

Despite the lack of quantitative results the following observations suggest that the proposed method shows a potential in supporting autistic children in learning imitation and turn taking behaviors at a very early age. (1) Most of the children managed to imitate the play scenarios. (2) The videos showed to be a suitable way to teach the children understand and imitate the target behavior. (3) The stress levels of the children stayed lower than in actual social contact with new person, as observed by the coordinator and children could get prepared well for the upcoming scenario.

Earlier studies have shown that the basic principles of this method, can improve the social skills of the children. We changed the setting by introducing multiagent system of tangible interaction blocks that were especially designed for autistic children and 
were tested to be perceived as very pleasurable and engaging. It is common for children with autism to choose the play objects based on the sensory stimulation that they provide, such as color, touch, sound, or smell. The i-blocks, which emit pleasant dimmed light, seen through the semi-transparent walls, has been well accepted by all autistic children that has been participating in the experiments so far. Implementing an accelerometer within the i-blocks makes them sensitive to handling with a hand and makes possible to implement and test imitation and other interaction behaviors that are rooted in motor handling actions. We are able to test in the future the ability of the children of mimicry or blind imitation, of goal imitation or of the overall process of imitation proper. At this study goal imitation was used to further facilitate turn taking. Both imitation and turn taking are components of cooperative play behaviors. We showed that when all the actions are well understood by the autistic children, they performed willingly turn taking behaviors, which they normally do not do.

We plan a longitudal study with an extended range of imitation and turn taking games that will aim at testing whether the children will be able to transfer the learned cooperative play behaviors to different, preferably real life situations. Improvements in quality and quantity of the videos will be necessary to keep the children interested on longer term. Within the current set-up the video is acted out by two amateur actors. For a final result the video scenarios need professional actors with prior experience in making children videos. These actors will be required to make the video perfectly understandable and enjoyable for the children. The set up should also be more professional regarding the lighting conditions of the room where the video material was taken and professional editing can also remove some minor flaws from the videos.

In the current research the play of a child and a caregiver in a prepared environment has been observed. We would like to explore the effect of this method in natural environment and in realistic every day interactions, such as at school with autistic and typical children.

\section{Acknowledgments}

We would like to thank Dr. Juliane Cuperus, Health psychologists/ neuropsychologist from Sint Marie hospital in Eindhoven, the Netherlands, for her evaluation collaboration. We also thank Rachel Delhees and Marleen Vissers for their collaboration setting up and carrying out the tests. Finally we thank Het Palet school in Weert, the Netherlands for their support.

\section{References}

[1] Aldridge, M.A., Stone, K.R., Sweeney, M.H., Bower, T.G.R.: Preverbal children with autism understand the intentions of others. Developmental Science 3, 294-301 (2000)

[2] Alers, S.H.M., Barakova, E.I.: Multi-agent platform for development of educa-tional games for children with autism. In: IEEE ICE CIG 2009, pp. 47-53 (2009) ISBN: 978-14244-4459-5

[3] Barakova, E.I., Gillessen, J., Feijs, L.: Social training of autistic children with interactive intelligent agents. Journal of Integrative Neuroscience 8(1), 23-34 (2009) 
[4] Barakova, E.I., van Wanrooij, G., van Limpt, R., Menting, M.: Using an emergent system concept in designing interactive games for autistic children. In: Proc. of IDC 2007, Aalborg Denmark, June 2007, pp. 73-77. ACM, New York (2007) 978-1-59593-747-6

[5] Barakova, E.I., Vanderelst, D.: From spreading of behavior to dyadic interaction-a robot learns what to imitate. Int. J. Intell. Syst. (2020) (in press)

[6] Barakova, E.I., Lourens, T.: Expressing and interpreting emotional movements in social games with robots. In: Personal and Ubiquitous Computing, pp. 1-11. Springer, Heidelberg (2010)

[7] Bekker, M.M., Sturm, J., Barakova, E.I.: Designing for social interaction through physical play. In: Sturm, J.A., Bekker, M.M. (eds.) Proc. Design for social interaction through physical play, Eindhoven, The Netherlands, October 22 2008, pp. 7-10 (2008)

[8] Bekker, T., Sturm, J., Eggen, J.: Designing Playful Interactions for social interaction and physical play. Personal and Ubiquitous Computing (2010), doi:10.1007/s00779-0090264-1

[9] Billard, A., Mataric, M.J.: Learning human arm movements by imitation: Evaluation of a biologically inspired connectionist architecture. Robotics and Autonomous Systems 37(23), 145-160 (2001)

[10] Breazeal, C., Buchsbaum, D., Gray, J., Gatenby, D., Blumberg, B.: Learning from and about others: towards using imitation to bootstrap the social understanding of others by robots. Artif. Life 11(1/2), 3-62 (2006)

[11] Charlop-Christ, M.H., Le, L., Freeman, K.A.: A Comparison of Video Modeling with In Vivo Modeling for Teaching Children with Autism. Journal of Autism and Developmental Disorders, 537-552 (2004)

[12] Corbett, B.A.: Video Modeling: A Window into the World of Autism. The Behavior Analyst Today 4(3) (2003)

[13] Corbett, B.A., Abdullah, M.: Video Modeling: Why Does It Work for Children with Autism? Journal of Early and Intensive Behavior Intervention 2(1), 2-8 (2005)

[14] D’Ateno, P., Mangiapanello, K., Taylor, B.A.: Using Video Modeling to Teach Complex Play Sequences to a Preschooler with Autism. Journal of Positive Behavior Interventions 5(1), 5-11 (2003)

[15] Escalona, A., Field, T., Nadel, J., Lundy, B.: Brief Report: Imitation Effects on Children with Autism. Journal of Autism and Developmental Disorders, 141-144 (2004)

[16] Feil-Seifer, D.J., Mataric, M.: Robot-Assisted Therapy for Children with Autism Spectrum Disorders. In: Proc. IDC 2008: Children with Special Needs, Chicago, USA (2008)

[17] Field, T., Field, T., Sanders, C., Nadel, J.: Children with autism display more social behaviors after repeated imitation sessions. Autism 5(3), 317-323 (2001)

[18] Jahr, E., Eldevik, S., Eikeseth, S.: Teaching children with autism to initiate and sustain cooperative play. Elsevier Science Inc., Amsterdam (2000)

[19] LeGoff, D.: Use of LEGO as a therapeutic medium. Journal of Autism and Developmental Disorders 34(5), 557-571 (2004)

[20] Lourens, T., Barakova, E.I.: My sparring partner is a humanoid robot. In: Mira, J., Ferrández, J.M., Álvarez, J.R., de la Paz, F., Toledo, F.J. (eds.) IWINAC 2009. LNCS, vol. 5602, pp. 344-352. Springer, Heidelberg (2009)

[21] Nadel, J.: Early imitation and a sense of agency. In: Proceedings of the 4th international workshop on epigenetic robots (2004)

[22] Nadel, J., Simon, M., Canet, P., Soussignan, R., Blancard, P., Canamero, L., Gaussier, P.: Human responses to an expressive robot. In: Proceedings of Epigenetic robotics 2006. Lund University Cognitive Studies, vol. 128, pp. 79-86 (2006) 
[23] Owens, G., Granader, Y., Humphrey, A., Baron-Cohen, S.: LEGO therapy and the social use of language programme. Journal of autism and developmental disorders 38(10), 1944-1957 (2008)

[24] Parten, M.B.: Social participation among pre-school children. Journal of Abnormal \& Social Psychology 27(3) (1932)

[25] Robins, B., Dautenhahn, K., Dickerson, P.: Robots as embodied beings-interactionally sensitive body movements in interactions among autistic children and a robot. In: Proc. of ROMAN 2005, pp. 54-59 (2005)

[26] Robins, B., Dickerson, P., Stribling, P., Dautenhahn, K.: Robot-Mediated Joint Attention in Children with Autism: A Case Study in Robot-Human Interaction. Interaction Studies 5(2), 161-198 (2004)

[27] Stanton, C.M., Kahn Jr., P.H., Severson, R.L., Ruckert, J.H., Gill, B.T.: Robotic Animals Might Aid in the Social Development of Children with Autism. In: Procs. ACM/IEEE Int. Conf. on Human-Robot Interaction, Amsterdam, Netherlands (2008)

[28] Thelen, M.H., Fry, R.A., Fethenbach, P.A., Frautsch, N.M.: Therapeutic videotape and film modeling: A review. Psychological Bulletin 86, 701-720 (1979)

[29] Want, S.C., Harris, P.L.: How do children ape? Applying concepts from the study of nonhuman primates to the developmental study of 'imitation' in children. Developmental Science 5(1), 1-14 (2009) 